\title{
A INCIDENCIA DOS DISTÚRBIOS OSTEOMUSCULARES JUNTO AO FUNCIONALISMO PÚBLICO NO SETOR DA SECRETARIA DE SAÚDE NA CIDADE DE LAURO MULLER- SC.
}

The incidence of osteomuscular disturbances with public functionality in the secretariat of health in the city of lauro muller-sc.

Tiago B. de Almeida ${ }^{1}$, Ralph F. Rosas ${ }^{2}$

${ }^{1}$ Fisioterapeuta Graduado pela Universidade do Sul de Santa Catarina (UNISUL), Pós Graduando em Fisioterapia Traumato-Ortopédica pela Universidade Extremo Sul Catarinense(UNESC)

${ }^{2}$ Fisisoterapeuta, Doutor em Ciência do Movimento pela Universidade do Sul de Santa Catarina(UNISUL), Docente e Coordenador do Curso de Fisioterapia na Universidade do Sul de Santa Catarina (UNISUL).

\section{Endereço para Correspondência}

Tiago Blasius de Almeida

Rua: Cairú-, 162 Bairro: Cairú

Lauro Muller-SC

Fone: (48) 3464-5093

Email: fisioclinicact@gmail.com 


\title{
RESUMO
}

Trabalhadores da saúde são todos aqueles que se inserem direta ou indiretamente na prestação de serviços de saúde, no interior dos estabelecimentos de saúde ou em atividades de saúde, podendo deter ou não formação específica para o desempenho de funções atinentes à área. Os distúrbios musculoesqueléticos são responsáveis por elevados índices de absenteísmo e incapacidade temporária ou permanente, com reflexos negativos também para a qualidade dos serviços de saúde prestados. No Brasil os Distúrbios Osteomusculares Relacionados ao Trabalho (ou Lesões por Esforços Repetitivos - LER/DORT) representam as doenças ocupacionais mais frequentes no país. Os distúrbios musculoesqueléticos são multicausais, eventualmente aparecendo de maneira lenta e insidiosa, e podem se desenvolver em função da exposição contínua e prolongada dos trabalhadores a efeitos nocivos e adversos no ambiente de trabalho. O objetivo do presente estudo é verificar a incidência dos distúrbios osteomusculares junto ao funcionalismo público no setor da secretária de saúde na cidade de Lauro Muller- SC. A população/amostra será composta por 23 funcionários em efetivo exercício na secretaria, pelo com pelo menos 6 meses de serviço. Após o consentimento, os dados serão obtidos através de um questionário autoaplicável, pelo QNSO para sintomas osteomusculares, a EVA para medir a dor dos desconfortos dos indivíduos e sintomas nas diversas regiões anatômicas, bem como um questionário sobre a percepção das condições de trabalho e suas relações com a condição de saúde.

Palavras-chave: LER. Dor musculoesquelética. Estudos de avaliação.

\begin{abstract}
Health workers are all those who are directly or indirectly involved in the provision of health services, within health facilities or in health activities, and may or may not have specific training for the performance of functions related to the area. Musculoskeletal disorders are responsible for high rates of absenteeism and temporary or permanent disability, with negative consequences for the quality of
\end{abstract}


health services provided. In Brazil, Work-related Musculoskeletal Disorders (RSI) are the most frequent occupational diseases in Brazil. Musculoskeletal disorders are multicausal, eventually appearing slowly and insidiously, and may develop as a result of continuous and prolonged exposure of workers to harmful and adverse effects in the work environment. The objective of the present study is to verify the incidence of musculoskeletal disorders in the public health sector in the city of Lauro Muller-SC. The population / sample will be composed of 23 employees in effective exercise in the office, for at least 6 months of service. After consent, the data will be obtained through a self-administered questionnaire, by the QNSO for musculoskeletal symptoms, VAS to measure the pain of the discomforts of individuals and symptoms in the various anatomical regions, as well as a questionnaire on the perception of working conditions and their relation to health status.

Keywords: READ. Musculoskeletal pain. Evaluation studies.

\section{INTRODUÇÃO}

Trabalhadores da saúde são todos aqueles que se inserem direta ou indiretamente na prestação de serviços de saúde, no interior dos estabelecimentos de saúde ou em atividades de saúde, podendo deter ou não formação específica para o desempenho de funções atinentes à área. O principal critério adotado na definição de quem são os trabalhadores da saúde é o vínculo de trabalho no setor, independente da formação do indivíduo'.

No campo de estudo das relações saúde e trabalho está proposto o modelo explicativo das iniquidades em saúde musculoesquelética, pois estão suficientemente descritas as desigualdades sociais no tocante à exposição aos fatores de risco conhecidos e aos seus efeitos mórbidos. Os distúrbios musculoesqueléticos são responsáveis por elevados índices de absenteísmo e incapacidade temporária ou permanente, com reflexos negativos também para a qualidade dos serviços de saúde prestados ${ }^{1}$.

A morbidade musculoesquelética vem se configurando no mundo inteiro como uma relevante questão de saúde pública, representando um alto custo social 
para o trabalho, podendo levar a aposentadorias. No Brasil, o (MPS) informa que os Distúrbios Osteomusculares Relacionados ao Trabalho (ou Lesões por Esforços Repetitivos - LER/DORT) representam as doenças ocupacionais mais frequentes no país ${ }^{2}$.

Os distúrbios musculoesqueléticos quando relacionados ao trabalho possuem implicações socioeconômicas graves nas sociedades modernas e são comuns entre os profissionais de saúde devido à sua exposição a vários riscos ocupacionais, tais como: inadequadas posturas, manuseio de materiais contaminados e níveis elevados de estresse no local de trabalho ${ }^{3}$.

Os distúrbios musculoesqueléticos são multicausais, eventualmente aparecendo de maneira lenta e insidiosa, e podem se desenvolver em função da exposição contínua e prolongada dos trabalhadores a efeitos nocivos e adversos no ambiente de trabalho ${ }^{5}$.

Inúmeras categorias profissionais são atingidas pelo agravo denominado LER/DORT, nomenclaturas adotadas pelo Ministério da Saúde que definem esses agravos como fenômenos relacionados ao trabalho. O principal agravo, responsável em quantidade de auxílios-doença e valor de auxílios-doença acidentários - cedidos pela Previdência Social entre 2011 e 2013 - são as doenças do sistema osteomuscular e do tecido conjuntivo ${ }^{4}$.

O acrônimo LER, no Brasil, alcançou o senso comum desde as negociações sociais nos anos 1980, pelo reconhecimento do caráter ocupacional dos distúrbios. Apesar de guardar imprecisões, DORT seria mais abrangente, uma vez que os sintomas podem surgir associados a um esforço ou trauma, e não exclusivamente ao gesto repetitivo como sugere o acrônimo que se tornou mais difundido. Vale ressaltar que fatores ambientais e tarefas que exigem repetição e posturas estereotipadas são riscos reconhecidos ${ }^{4}$. Os seus sintomas podem se apresentar isolada ou concomitantemente, destacando-se, principalmente, dor e desconforto em pescoço, ombros, região cervical, região lombar, e membros inferiores ${ }^{5}$.

Além de não saudável, o trabalho é difícil e repetitivo e pode provocar lesões físicas irreversíveis; profissionais são vítimas de distúrbios 
músculoesqueléticos que podem resultar em esgotamento, relacionado à sua exposição a fatores fisiológicos encargos, acompanhados de sintomas dolorosos ${ }^{9}$.

Os mais atingidos são os jovens, os pouco qualificados, do sexo feminino, geralmente a faixa etária entre 20 a 40 anos estão mais propensas ao desenvolvimento das lesões musculares por movimentos repetitivos, pois os tendões e ligamentos femininos são mais frágeis do que o dos homens, além do mais, a mulher une a jornada de trabalho a suas atividades domesticas, se sobrecarregando cotidianamente $^{10}$.

De acordo com as normas técnicas do MPS, a sintomatologia apresentada nos DORT costuma englobar dor, parestesia, sensação de peso e fadiga, além de abranger quadros clínicos do sistema musculoesquelético adquiridos pelo trabalhador submetido a determinadas condições de trabalho. A presença desses sintomas é fator preponderante para uma percepção negativa do indivíduo em relação à sua saúde ${ }^{6}$.

Nessa perspectiva, a mensuração dos relatos de DORT faz-se necessária afim de que se tenham dados para quantificar as prevalências e avaliar diversas populações acometidas. Dentre as ferramentas utilizadas para avaliação dessa sintomatologia, o Questionário Nórdico de Sintomas Osteomusculares (QNSO) é um instrumento desenvolvido com a proposta de padronização dos relatos de dores e desconfortos no sistema musculoesquelético, facilitando assim a comparação entre resultados de diversos estudos e populações ${ }^{6}$.

O instrumento é representado por uma figura de um corpo humano, dividido em nove regiões anatômicas: pescoço, ombros, região dorsal e lombar das costas, cotovelos, punhos/mãos, quadril/coxas, joelhos, tornozelos/pés, não sendo utilizada para avaliação a região do antebraço; constitui-se de alternativas múltiplas e binárias, as quais deverão ser respondidas quanto à presença ou não dos sintomas musculoesqueléticos nas diferentes estruturas corporais já mencionadas ${ }^{7}$.

Além do QNSO, será utilizada a escava visual analógica (EVA) para medir a dor dos desconfortos dos indivíduos avaliados. A EVA é um instrumento unidimensional para a avaliação da intensidade da dor. Trata-se de uma linha com as extremidades numeradas de 0-10. Em uma extremidade da linha é marcada 
"nenhuma dor" e na outra "pior dor imaginável". Pede-se, então, para que o paciente avalie e marque na linha a dor presente naquele momento ${ }^{8}$.

O objetivo do presente estudo é verificar a prevalência dos distúrbios osteomusculares junto ao funcionalismo público no setor da Secretária de Saúde na cidade de Lauro Müller - SC, aplicando o QNSO e EVA.

\section{METODOLOGIA}

Trata-se de um estudo descritivo de corte transversal e quantitativo. A população em estudo foi constituída por 23 funcionários públicos municipais da secretaria de saúde de Lauro Muller-SC; foram utilizados como critérios de inclusão funcionários em efetivo exercício na secretaria, pelo com pelo menos 6 meses de serviço, e como critério de exclusão funcionários afastados, aqueles que apresentam transtornos neurológicos graves, patologias ortopédicas graves e aqueles que apresentam problemas em membros inferiores bem como aqueles que não aceitaram participar.

Para isto aplicou-se o Questionário Nórdico para levantamento do segmento corporal e EVA para intensidade de dor pontuada de zero a 10, onde zero compreende a ausência de dor e dez a pior dor imaginável, sendo categorizadas em leve (1, 2 e 3), moderada ( 4,5 e 6 ) e grave (7, 8, 9 e 10), permitindo uma pontuação precisa da dor, sendo também de rápida e fácil aplicabilidade.

Os dados obtidos através do questionário QNSO foram analisados estatisticamente a partir do software IBM SPSS Statistics versão 20.0. Os resultados serão expressos por meio de frequências. A EVA foi analisada por meio de estatística descritiva (média, desvio padrão e afins). As análises inferenciais foram realizadas com nível de confiança de $95 \%$.

A distribuição das variáveis quanto à normalidade foi avaliada por meio dos testes de Shapiro-Wilk.

Adotou-se como variáveis para estudo dor e região corporal.

\section{RESULTADOS}


Este estudo teve como objetivo verificar a prevalência dos distúrbios osteomusculares nos funcionários da Secretária de Saúde na cidade de Lauro Müller. Foram avaliados 23 funcionários.

Dentro da população pesquisada, verificou-se que a prevalência de desconforto postural foi maior, em ordem crescente, nas seguintes regiões: lombar $(n=8)$, ombros $(n=6)$, coluna torácica $(n=3)$, cabeça $(n=2)$, coluna cervical $(n=2)$, punhos $(n=1)$ e pernas $(n=8)$. As regiões outras regiões corporais não foram citadas. Os dados podem ser melhor visualizados no Gráfico 1 abaixo.

\section{Gráfico 1 - Regiões anatômicas investigadas no Questionário Nórdico de Sintomas Osteomusculares.}

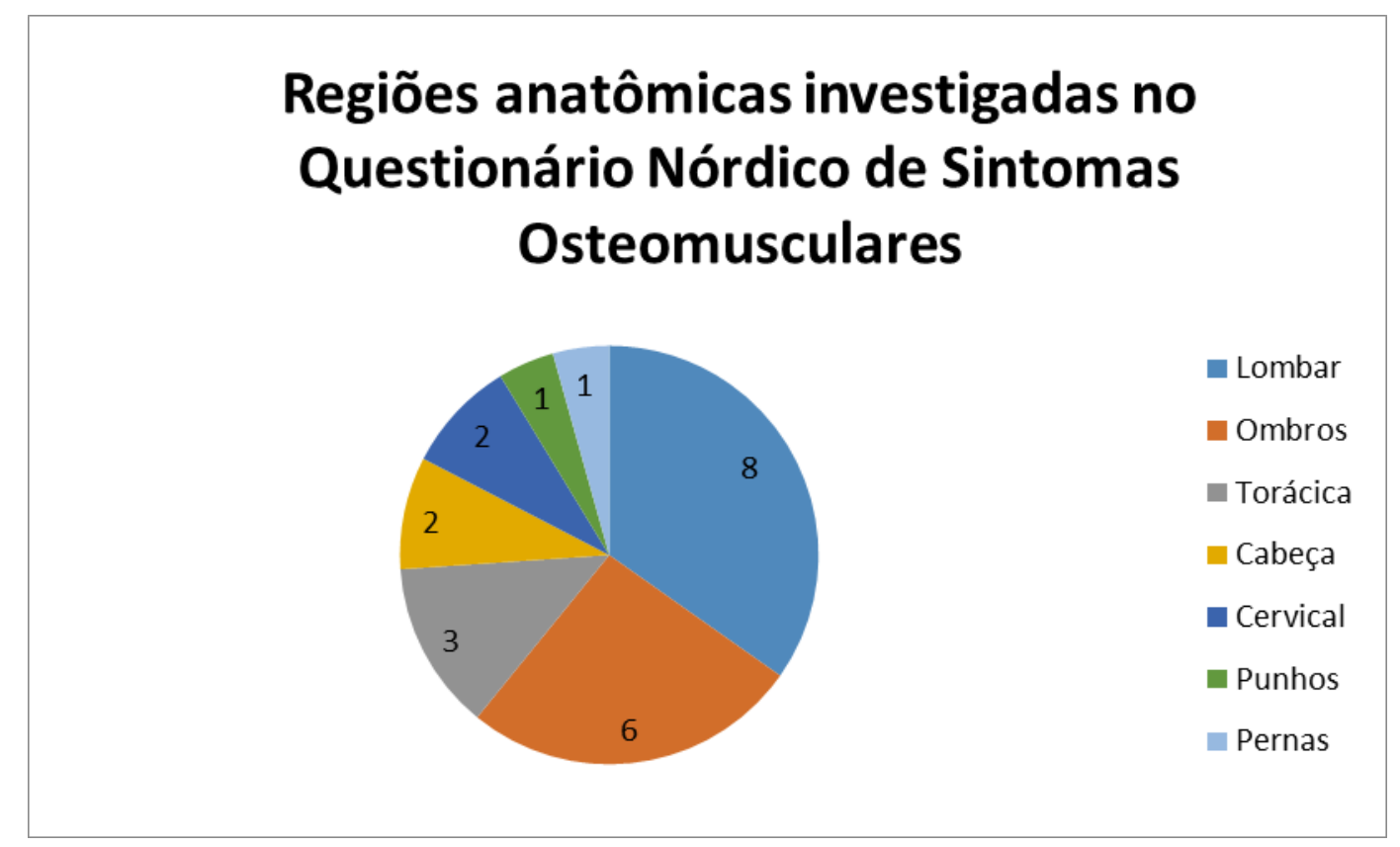

Fonte: dados coletados pelos autores (2018).

Ainda se verificou a intensidade da dor através da EVA, em centímetros, tendo como média geral $6,81( \pm 1,77)$. Um funcionário referiu EVA máxima 10 (na cabeça) e a menor dor observada foi coluna torácica por um indivíduo apenas (EVA 3). O teste de Shapiro-Wilk $(\alpha=0,05)$ demonstrou que a amostra era normal/paramétrica. A estatística descritiva dos dados encontrados em relação à dor está disposta na tabela abaixo. 
Artigo Original

Fisiopatologia

\begin{tabular}{ll}
\hline Tabela 1 - Estatística descritiva da EVA. & \\
\hline Média & 6,81 \\
Idade [IC 95\%] & {$[5,84-7,37]$} \\
Idade p unilateral* $^{*}$ & 0,49 \\
Idade p bilateral* $^{*}$ & 0,99 \\
Desvio-padrão & 1,77 \\
Mediana & 7 \\
Mínimo & 3 \\
Máximo & 10 \\
Moda & 8 \\
Amplitude & 7 \\
Curtose & $-0,57$ \\
Distorção & $-0,24$ \\
1ํquartil & 5 \\
3o quartil & 8 \\
Shapiro-Wilk\# & 0,94 \\
\hline
\end{tabular}

Valores expressos em centímetros.

$I C=$ intervalo de confiança $(\alpha=0,05)$.

* $p>0,05$ ao teste t para uma amostra.

$\# p>0,05$ ao teste de Shapiro-Wilk.

\section{DISCUSSÃO}

O presente estudo mostrou maior prevalência de distúrbio osteomuscular na região de lombar $35 \%$ e ombros $26 \%$, da população pesquisada, os demais distúrbios foram encontrados nos segmentos cabeça $9 \%$, cervical $9 \%$, punhos/mãos $4 \%$, pernas $4 \%$.

Os resultados obtidos são convergentes com alguns estudos; em estudo realizado com trabalhadores de enfermagem de um hospital universitário do Rio Grande do Sul verificou que, no último ano, as estruturas anatômicas mais acometidas pela dor ou desconforto foram a região lombar das costas $(71,5 \%)$, pescoço $(68 \%)$, ombros $(62,2 \%)$ e pernas $(54,6 \%)$. Dentre as regiões corporais que 
obtiveram maior percentual quanto a impedir o desenvolvimento das atividades diárias, houve destaque para a região lombar das costas $(60,4 \%)$, pulsos/mãos (58\%), região cervical das costas $(54,7 \%)$ e cotovelos $(54,1 \%)$. Nos últimos sete dias, as prevalências foram na região lombar das costas $(56,4 \%)$, pernas $(49,6 \%)$ e pescoço $(47,9 \%)^{16}$

Um estudo desenvolvido com profissionais de enfermagem de 11 hospitais da cidade de Londrina/PR constatou a presença dos sintomas musculoesqueléticos nos últimos 12 meses, na região lombar das costas (38,9\%) e ombros (37,9\%). Ainda destacou-se que a região lombar das costas $(11,4 \%)$ obteve o maior percentual perante o impedimento para realização de atividades diárias. Nos últimos sete dias, a estrutura mais acometida foi a região lombar das costas $(20,4 \%)^{17}$

Os distúrbios do ombro são influenciados por fatores biomecânicos relacionados ao trabalho, como flexão ou abdução dos ombros por tempo prolongado, vibrações, postura estática ou com carga no membro superior. Uma relação do tipo dose/resposta entre sobrecarga e doenças do ombro foi encontrada nos trabalhos. Os resultados de alguns dos estudos evidenciam associação entre os distúrbios do ombro e os fatores psicossociais como estresse, longas jornadas de trabalho, período de descanso insatisfatório ${ }^{12}$.

Alguns estudos revelam que a prevalência das queixas autorreferidas ao ombro está estimada entre 16 e $26 \%$, ressaltam que a tendinite do manguito rotador, a tendinite da longa porção do bíceps braquial e a bursite subacromial constituem as principais causas de dor referida ao ombro ${ }^{13,14}$, a qual está associada à diminuição do estado de saúde e da qualidade de vida, sendo desejável o desenvolvimento de medidas para a sua prevenção primária ou secundária. A principal característica clínica sugestiva de patologia periarticular do ombro é o ritmo inflamatório da dor.

Um estudo realizado em 2012 revelou que, nos doentes com diagnóstico de lesão manguito rotador unilateral, a prevalência de casos assintomáticos no membro superior contralateral era de $35,5 \%$. Um número considerável de doentes assintomáticos desenvolveu sintomatologia passado um curto período de tempo do acompanhamento e apresentou deterioração da função da articulação do ombro ${ }^{11}$.

$O$ estudo identificou alta prevalência de lombalgia entre os indivíduos que executam suas atividades laborais sentados. A postura sentada mantida por tempo 
prolongado pode gerar carência de flexibilidade muscular e de mobilidade articular, além de fadiga dos músculos extensores espinhais que, aliados, comprometem a estabilidade e o alinhamento da coluna vertebral. Tais distúrbios biomecânicos são considerados importantes fatores etiológicos para o desenvolvimento de dor lombar ${ }^{13}$.

Como a amostra desta pesquisa trabalha na maior parte do tempo sentada, é possível explicar tamanha prevalência de dor na região lombar. Acredita-se que a postura sentada e 0 sedentarismo possam provocar alterações musculoesqueléticas. $\mathrm{O}$ uso excessivo da cadeira pode ocasionar um encurtamento gradual dos músculos iliopsoas e isquiostibiais, diminuindo a mobilidade da articulação do quadril e inclinando para frente o segmento lombar da coluna vertebral ${ }^{14}$. Além disso, essa posição pode causar fadiga e sobrecarga nos elementos passivos articulares (cápsulas, ligamentos e discos intervertebrais), ocasionando dessensibilização dos mecanoceptores teciduais e consequente redução ou eliminação da força estabilizadora profunda, gerada principalmente pelo multifídio lombar e transverso abdominal ${ }^{13}$.

É importante o reconhecimento do ambiente de trabalho antes da implantação de qualquer programa de intervenção, seja ele preventivo e/ou terapêutico, além do acompanhamento regular dos projetos executados para estimular mudanças de comportamento em longo prazo ${ }^{15}$.

\section{CONCLUSÃO}

Os resultados apresentados sugerem uma provável associação entre dor lombar e dor em ombros com as atividades laborais pela população pesquisada alertam para a necessidade de cuidados com a saúde dos mesmo no ambiente de trabalho, a qual sugere-se que sejam implantados programas de prevenção a saúde dos trabalhadores bem como estudos mais detalhados na área de ergonomia no setor pesquisado. 


\section{REFERÊNCIAS}

1. BARBOSA, Rose Elizabeth Cabral; ASSUNÇÃO, Ada Ávila; ARAÙJO, Tânia Maria de. Distúrbios musculoesqueléticos em trabalhadores do setor saúde de Belo Horizonte, Minas Gerais, Brasil. Cadernos de Saúde Pública, Rio de Janeiro, v. 28, n. $8, \quad$ p.1569-1580, ago. 2012. FapUNIFESP (SciELO). http://dx.doi.org/10.1590/s0102-311x2012000800015. Disponível em:< http://www.scielo.br/scielo.php?script=sci_arttext\&pid=S0102-311X2012000800015> . Acesso em 19 out. 2017.

2. FERNANDES, Rita de Cássia P.. Precarização do trabalho e os distúrbios musculoesqueléticos. Caderno Crh, Salvador, v. 24, n. 1, p.155-170, 2011. FapUNIFESP (SciELO). http://dx.doi.org/10.1590/s0103-49792011000400011. Disponível em: $\quad<\quad$ http://www.scielo.br/scielo.php?pid=S010349792011000400011\&script=sci_abstract\&tIng=pt $>$. Acesso em: 23 out.2017.

3. GARBIN, ArtÊnio JosÉ Ísper et al. Musculoskeletal disorders and perception of working conditions: A survey of Brazilian dentists in São Paulo. International Journal Of Occupational Medicine And Environmental Health, Sao Paulo, p.367377, 18 dez. 2017. Nofer Institute of Occupational Medicine. http://dx.doi.org/10.13075/ijomeh.1896.00724. Disponivel em: < https://www.ncbi.nlm.nih.gov/pubmed/28481371>. Acesso em: 23 out. 2017.

4 VIEGAS, Louise Raissa Teixeira; ALMEIDA, Milena Maria Cordeiro de. Perfil epidemiológico dos casos de LER/DORT entre trabalhadores da indústria no Brasil no período de 2007 a 2013. Revista Brasileira de Saúde Ocupacional, Salvador, v. 41, p.2317-6369, 2016. FapUNIFESP (SciELO). http://dx.doi.org/10.1590/23176369000130615. Disponivel em: <http://www.scielo.br/scielo.php?pid=S0303-

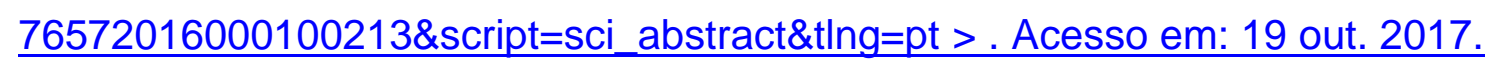




\section{Fisiopatologia}

5. ASSUNÇÃO, Ada Ávila; ABREU, Mery Natali Silva. Factor associated with self-reported work-related musculoskeletal disorders in Brazilian adults. Revista de Saúde Pública, [s.l.], $\quad$ v. $\quad 51, \quad$ n. $1,2017 . \quad$ FapUNIFESP (SciELO). http://dx.doi.org/10.1590/s1518-8787.2017051000282. Disponível em: $<$ http://www.scielo.br/scielo.php?pid=S0034$89102017000200301 \&$ script=sci_arttext\&tlng=pt $>$. Acesso em: 23 out. 2017.

6. LIMA JÚNIOR, José Pereira de; SILVA, Tarcísio Fulgêncio Alves da. Analysis of musculoskeletal disorders symptoms in professors of the University of Pernambuco - Petrolina Campus. Revista Dor, Sao Paulo, v. 15, n. 4, p.1806-0013, dez. 2014. GN1 Genesis Network. http://dx.doi.org/10.5935/1806-0013.20140060. Disponível em: < http://www.scielo.br/scielo.php?pid=S1806-

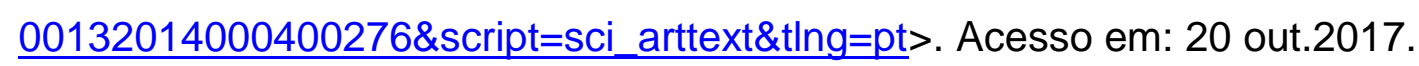

7 OLIVEIRA, Matheus Antochevis de et al. Distúrbios/dor musculoesquelética em estudantes de enfermagem de uma universidade comunitária do sul do Brasil. Enfermería Global, Rio Grande do Sul, v. 16, n. 3, p.145-159, 28 jun. 2017. Servicio de Publicaciones de la Universidad de Murcia. http://dx.doi.org/10.6018/eglobal.16.3.248551 Disponivel em $\quad:<$ http://revistas.um.es/eglobal/article/viewFile/248551/212931>. Acesso em : 24 out. $\underline{2017 .}$.

8. MARTINEZ, José Eduardo; GRASSI, Daphine Centola; MARQUES, Laura Gasbarro. Análise da aplicabilidade de três instrumentos de avaliação de dor em distintas unidades de atendimento: ambulatório, enfermaria e urgência. Revista Brasileira de Reumatologia, Sao Paulo, v. 51, n. 4, p.304-308, ago. 2011. Elsevier BV. http://dx.doi.org/10.1590/s0482-50042011000400002. Disponível em: < http://www.scielo.br/scielo.php?pid=S0482-

50042011000400002\&script=sci_abstract\&tIng=pt $>$. Acesso em : 20 out.2017. 
9. LELIS, Cheila Maíra et al. Distúrbios osteomusculares relacionados ao trabalho em profissionais de enfermagem: revisão integrativa da literatura. Acta Paulista de Enfermagem, São Paulo, v. 25, n. 3, p.477-482, jan. 2012. FapUNIFESP (SciELO). http://dx.doi.org/10.1590/s0103-21002012000300025 Disponível em http://www.scielo.br/scielo.php?pid=S0103-

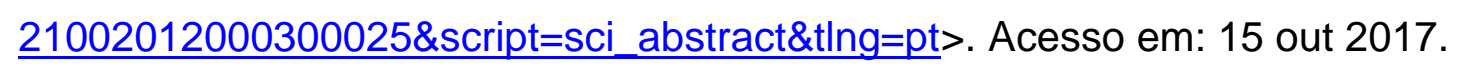

10. RAGADALI FILHO, Alvaro. Lesões Por Esforços Repetitivos (Ler): Uma Doença Misteriosa Do Trabalho. Rev. Saberes, São Paulo, v. 4, n. 1, p.2358-0909, jan. 2016.Disponível em: <http://facsaopaulo.edu.br/media/files/35/35 1386.pdf> . Acesso em: 26 de out. de 2017.

11. SILVEIRA, R,S. et al. Tendinite do manguito rotador em operadores de caixa de supermercado: contributos para a vigilância de saúde. Rev Bras Med Trab. 2017;15(2):158-66, Disponivel em: <http: www.rbmt.org.br/exportpdf/225/v15n2a06.pdf>. Acesso em 25 de março de 2018.

12. MENDONÇA JR H. P., ASSUNÇÂO, A. A. Associação entre distúrbios do ombro e trabalho: breve revisão da literatura. Rev Bras Epidemiol 2005; 8(2): 16776.Disponível em: < httpp: www.rbmt.org.br/export-pdf/225/v15n2a06.pdf> Acesso em 25 de março de 2018.

13. PANJABi M,M. A hypothesis of chronic back pain: ligament subfailure injuries lead to muscle control dysfunction. Eur Spine J 2006;15(5):668-76.

14. SACCO I.C.N, ALIBERTI S, et al. A influência da ocupação profissional na flexibilidade global e nas amplitudes angulares dos membros inferiores e da lombar. Rev Bras Cineantropom Desempenho Humano 2009; 11(1):51-8. Disponível em: <http:bases.bireme.br/cgi-bin/wxislind.exe/iah/online/?lsisScript=iah>. Acesso em: 25 março de 2018. 
Artigo Original

Fisiopatologia

15. BARROS, S. S., et al. Lombalgia ocupacional e a postura sentada. Rev Dor, São Paulo, 2011 jul-set;12(3):226-30. Dispnivel em: http:www.scielo.br/scielo.php?pid=S1806>. Acesso em: 25 de março de 2018.

16. Magnago TSBS, Lisboa MTL, Griep RH, Kirchhof ALC, Guido LA. Aspectos psicossociais do trabalho e distúrbio musculoesquelético em trabalhadores de enfermagem. Rev. Latino-Am. Enfermagem, 2010; 18(3) Disponível em: http:www.scielo.br/scielo.php?pid>. Acesso em: 25 de março de 2018.

17. Schmidt DRC, Dantas RAS. Qualidade de Vida no Trabalho e Distúrbios Osteomusculares Relacionados ao Trabalho entre profissionais de enfermagem. Acta paul. Enferm [Internet], 2012; 25(5): 701-707. Disponível em: http://www.scielo.br/scielo.php?pid=S0103-21002012000500009\&script=sci arttext. Acesso em 26 março 2018. 